\title{
ASYMMETRICAL CHARGE DISTRIBUTION OVER THE STRAINED SILICON MEMBRANE
}

\author{
Shobha Kanta Lamichhane \\ Associate Professor,Tribhuvan University, Prithvi Narayan Campus, Pokhara,
}

\begin{abstract}
It is experimentally proved that pressurized silicon membrane has heterogeneous distribution of force (i.e., pull-off force) along a line over the entire surface. Based on pull-off force, paper has shown surface energy, net strength of interaction and electrical conductivity along with topographical variation exist in an extended silicon membrane. For a sufficiently thin membrane that undergoes beyond the critical applied pressure get strained layer by layer. As a result, thickness of the strained layer increases that influence number of atoms and hence formation of lattice distortion. on continuation, at some point lattice-mismatch will occur which is reflected either in topography or in force spectroscopy or in surface energy distribution or in XRD or even in tunneling spectroscopy. Once again, generation of lattice mismatch result lattice distortion and localized states in membrane surface, means defects are not uniformly distributed hence generate heterogeneity in morphology. As a result asymmetrical charge distribution is occurring in membrane surface. These results indicate that for a critical applied pressure that induces an abrupt transition by which membrane is losing its elastic behavior and turn down to plastic regime, which is a concept that can be applied to macroscopic as well as microscopic systems. Macroscopically, the interfacial energy can be determined by contact angle measurements. For microscopic systems and even at molecular levels, its numerical value is close to that of a planer macroscopic surface composed of the same molecules.
\end{abstract}

Keywords: asymmetry;strained, heterogeneity; AFM; SFM; pull off force.

\section{INTRODUCTION}

The periodicity of the lattice should be sufficiently uniform to provide a symmetrical carrier distribution in bulk state. Real crystals have surface imperfections and impurities. So that solid like silicon can be considered as a collection of ions and loosely bind electrons or covalent crystals. Ions are very heavy objects and electrons are small. Thus the position of ions determines the crystal structure. Sometimes we chose to consider only the behaviour of the ions. It is convenient to separate a solid into ions and valence electrons. Ions are spread out to form a uniform background of positive charge through which the outer valence electrons move. Ions at or near the surface do not have the sufficient number of neighbours to satisfy their bonding. This results lattice distortion and then an asymmetrical charge distribution in the material adjacent to the surface. The result is that localized states in the surface and act as trap because of the incomplete bonding. For a sufficiently thin silicon membrane that undergoes beyond the critical applied pressure get strained layer by layers. As a result strained layer thickness increases. As the strained layer thickness increases, the total number of atoms under strain or the distorted atomic bound grows and at some point misfit lattices (i.e., mismatch) and defects are nucleated, as a result heterogeneity in morphology comes into picture.

Surface roughness and environment play a critical role in adhesion. As the surface approaches nanoscale dimension, the surface roughness and area of contact should reach comparable dimension, such that apparent and true area of contact becomes approximately equal. This is in favor of AFM nano-adhesion experiment. Physical structures used in technological applications have been reduced in size, there has been increasing need to understand the limiting processes [1] as such.

A number of forces comes into picture as the tip-sample distance becomes smaller and smaller. When surfaces are separated at an almost atomic distance, number of forces that act between two surfaces can cause them to adhere, which produces bond strengths comparable to form covalent/metallic bonding. At particular distance (i.e., fraction of nanometer) the surface plasma will provide a 
screening action, at the same time the metallic/hydrogen bond will begin to develop. The forces required to separate the two surfaces is adhesive or pull-off force.

When the surfaces (i.e., AFM tip-sample) are at an atomic distance apart, the full hydrogen bind will have been formed and the short-range repulsive forces comes into operation to provide final equilibrium between tip and the sample surface. The magnitude of such forces depends on the true contact area and nature of the attractive forces holding the surface together [1]. The nature of these forces could be van der Waals, electrostatic, chemical-bonding forces etc that are active across sample interface. Interaction forces and their effective ranges in SFM are listed in table1.

Table1: Interaction forces and their range in SFM

\begin{tabular}{ll}
\hline Type of force & Range (nm) \\
\hline Electrostatic & 100 \\
Double layer in electrolyte & 100 \\
Van der Waals & 010 \\
Surface induced solvent ordering & 005 \\
Hydrogen bonding & 00.2 \\
Contact & 00.1 \\
\hline
\end{tabular}

Source: Ref: [2]

The dominant interaction that exists between atoms, between molecules and between particles is van der Waals force. An atom is composed of a positively charged core and a surrounding negatively charged electron cloud. Statistically, it is conceivable that the nucleus and its electron cloud are momentarily displaced with respect to each other. This configuration constitutes an electric dipole. A neighboring atom senses this electric dipole and responds to it with a similar charge distribution. Two adjacent dipoles are then attracting each other. Thus, van der Waals forces are generated at the instant that an electron cloud density occurs at one side of an atom during the electron flight about the nucleus.

In fcc crystal like $\mathrm{Si}$, the valence electrons are shared by twelve neighbors leading to covalent bond in its bulk state. As the distance becomes closer and closer, bonds are down to metallic/hydrogen and van der Waals force dominates over the others. This is a kind of scaling effect [3] where the structure and energies of silicon may take many different configurations. In this line, bond formalism behaviors in strained silicon membrane are non-stationary, which is purposed to determine/identify by pull-off/adhesion portion of force curve generated by AFM.
Surface roughness and working environment play a critical role in adhesion. As the surface approaches nanoscale dimension, the surface roughness and area of contact should reach comparable dimension [1], such that apparent and true area of contact becomes approximately equal. Taken into account, AFM nano-adhesion experiment is increasingly popular to understand the micro-contact systems.

Number of forces comes into picture as the tip-sample distance becomes smaller and smaller. At particular distance (i.e., fraction of nanometer) the surface plasma provides a screening action, at the same time surface adhesion begins to develop. When surfaces are at an almost atomic distance, number of forces that act between two surfaces can cause them to adhere. The force required to separate the two surfaces is adhesive or pull-off force.

When the surfaces (i.e., AFM tip-sample) are at an atomic distance, the short-range repulsive forces comes into operation to provide final equilibrium between tip and the sample surface. The magnitude of such forces depends on the true contact area and nature of the attractive forces holding the surface together [1]. The nature of these forces could be van der Waals (vdW), Interaction forces and their effective ranges in SFM are listed in ref. [2].

An atom is composed of a positively charged core and surrounding negatively charged electron cloud. Statistically, it is conceivable that the nucleus and its electron cloud are momentarily displaced with respect to each other. This configuration constitutes an electric dipole. A neighboring atom senses this electric dipole and responds to it with a similar charge distribution. Two adjacent dipoles are then attracting each other. Thus, vdW forces are generated at the instant that an electron cloud density occurs at one side of an atom during the electron flight around the nucleus.

In an AFM experiment, when probe going down and touching the sample, the distance between them is in order of nanometer. At this range, vdW force dominates over others. As the distance is reduced further, the electrostatic force of repulsion is account into the picture. Measurements of pull-off force in AFM force spectroscopy consist of mainly vdW force. Interaction strength depends on the distance between two atoms [3]. The paper discuss about the net force of attraction in strained silicon membrane which is asymmetrically distributed as determine/identify by pull-off/adhesion portion of force curve generated by AFM. The paper also describes the structure and energy configuration of strained silicon membrane. 


\section{EXPERIMENTAL PROCEDURE}

Employing an anisotropic $\mathrm{KOH}$ etching, in p-type mirror polished silicon chip with resistivity $4 \Omega$-cms, produces a $27 \mu \mathrm{m}$ thick silicon smple was prepared and mounted on a specially designed pressure cell. Argon gas was used to pressurize the cell, which retains the gas pressure with the help of a self-activating valve. For the experiment reported here, the cell was pressurized to 3 bars. Employing an AFM from TM, USA, in contact mode, force curve measurements have been carried out. The pressurized cell is mounted on the AFM scanner to perform scans. The pull-off force reported here was recorded by symmetrically moving the sample on the $\mathrm{X}-\mathrm{Y}$ stage of the head of the AFM scanner. The force constant of the cantilever employed is $0.26 \mathrm{~N} / \mathrm{m}$. The instrument was automatically programmed to collect force distance curves (from which the pull-off force data was retrieved) from 16 points along a line.

XRD measurements were performed by the Philips X'pert, Holland with $\mathrm{Cu}-\mathrm{K} \alpha$ radiation for $45 \mathrm{KV}$ of anode voltage and $40 \mathrm{~mA}$ current at each stage of the processing. X-rays of wavelength $1.54{ }^{\circ} \mathrm{A}$ and a scanning speed of $2^{0}$ per min were chosen for all XRD measurements. Pressure cell with extended silicon membrane (i.e., pressurized) was placed on sample holder with some special arrangements. Keeping in mind that sample plane is horizontally adjusted such that there is no any disturbance for incoming X-rays and detector during the measurements.

\section{RESULT AND DISCUSSION}

AFM real space images of $(5 \mu \mathrm{m} \times 5 \mu \mathrm{m})$ and $(0.5 \mu \mathrm{m} \times$ $0.5 \mu \mathrm{m})$ scan size taken from a stressed membrane are display in Fig.1 (A) and (B). It is obvious from these images that Fig. (B) looks flat where as Fig.(A) has sequence of depression-elevation-depression-elevation. In order to observe such sequence of depressionelevation, we have taken small size images as display in Fig.2. Fig.2 (a) and (b) belong to AFM images of the same stressed silicon membrane of scan size $(1 \mu \mathrm{m}$ $\mathrm{x} 1 \mu \mathrm{m})$ each, for without-pressure and with-pressure along with their 3Ds. We have collected 10 such scans in different locations of the extended silicon membrane and a similar sequence of elevation-depression-elevation is observed.
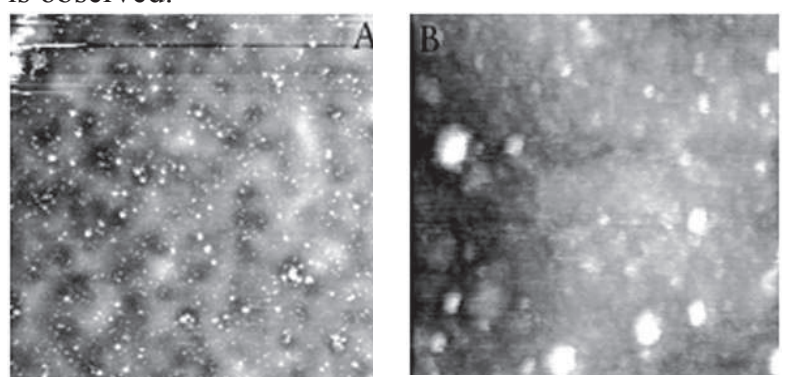

Fig 1: AFM mocrogrphs of pressurized silicon membrane of scan size (A) $5.0 \mu \mathrm{m} \times 5.0 \mu \mathrm{m}$, (B) $0.5 \mu \mathrm{m} \times 0.5 \mu \mathrm{m}$

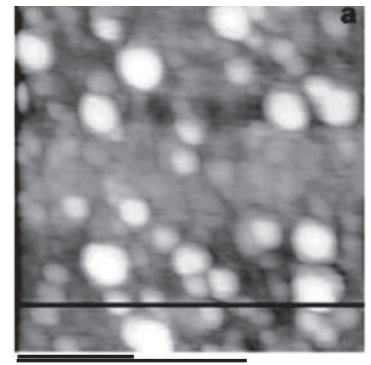

$500 \mathrm{~nm}$

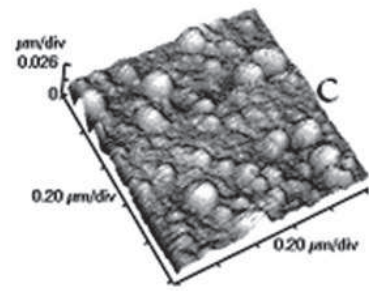

Fig 2: AFM micrograph of silicon membrane of scan size size ( $1 \mu \mathrm{m}$ $\times 1 \mu \mathrm{m}$ ) for (a) pressure less and (b) pressurized, membrane and their corresponding 3-Ds.

In our experiment the spatial mapping of pull-off force shown in Fig.3a communicates large pull-off force on either side of the middle at the depressions in image shown in Fig 2b. Fig.3a, shows the pull-off force data obtained on the silicon membrane, which has been deformed following application of a pressure of 3 bar and compare this with the pull-off force data from the same membrane prior to the application of pressure. Such non-linear force profile is explained on the basis of $\mathrm{vdW}$ force between the tip and the sample. In order to understand net strength of vdW force in our case, let's first discuss about how we achieve the surface energy and hence single atomic interaction between tip-sample based on collected pull-off force (shown in Fig 3a). Experimentally retrieved pull-off force data and some other processing data display in Table 2.

Micro-contact between AFM tip and the sample surface can be considered as adhesion. Adhesive force, connected with the interfacial energy, is a concept which can be applied to macroscopic well as microscopic systems [4]. Macroscopically, the interfacial energy can be determined by contact angle measurements. For microscopic systems and even at molecular levels, its numerical value is close to that of a planer macroscopic surface [5] composed of same molecules.

The work of adhesion, $\mathrm{W}_{132}$ (i.e., work required to separate surface 1 and surface 2 in medium 3, per unit area), is related to interfacial energy $[2,4]$ by

$W_{132}=\gamma_{13}+\gamma_{23}-\gamma_{12}$

Where $\gamma$ represents interfacial energy; 1,2 and 3 refer to sample surface, tip surface and contacting medium. If the measurements are performed in a vacuum, the interfacial energy $\gamma_{B}$ is simply surface free energy, $\gamma_{1}$ 


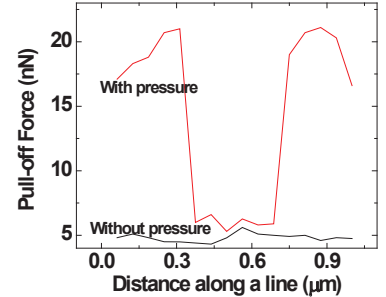

(a)

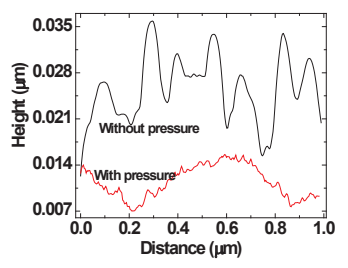

(b)

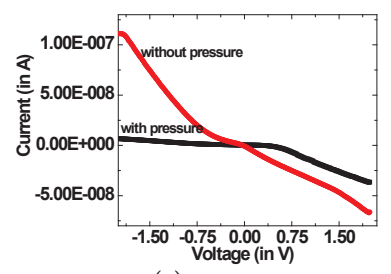

(c)

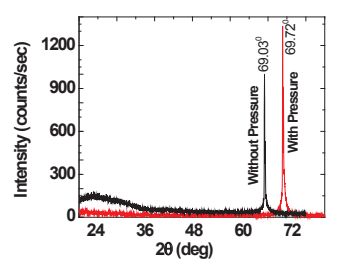

(d)

Fig 3: Results of without pressure and with pressure conditions(a) pull-off force distribution (b) roughness distribution (c) IV characteristics and (d) XRD spectra

In such a case surface energy and work of adhesion can be correlated. But, in other cases, when measurements are performed between symmetric contacts (i.e., $\left.\gamma_{1}=\gamma_{2}\right)[2,6]$ in medium 3 , then

$W_{132}=2 \gamma_{13}$

The pull-off force under Lennard-Jones potential [7] that can be seen micro contact between the tip and sample surface $[9,4]$ is

$$
F_{\text {pull-off }}=\frac{3}{2} \pi \mathrm{RW}_{123} \quad \text { Where } \frac{1}{R}=\frac{1}{R_{1}}+\frac{1}{R_{2}}
$$

$R$ is effective radius of radii $R_{1} \& R_{2}$. In AFM, $R_{1} \& R_{2}$ belongs to probe and sample radius. But, $R_{2}$ is infinite, so, $R \cong R_{1}$, the tip radius.

This formulation provides a basis for relating the work of adhesion and interfacial energies to adhesion force obtained by micro-contact rupture from pull-off portion of the curve.

JKR (Johnson-Kendall-Robert) theory [7] considers that short-range interfacial forces are operative and that finite contact areas exist upon rupture. When micro-contact ruptures, the concept of surface energy, which relates adhesive energy, can be estimated. AFM measures adhesive forces that can be used to estimate the number of molecular contacts at instant of rupture. The detail of this proposition is discussed below. Let's begin our discussion with contact radius. In JKR theory [7, 8], the contact radius is

$$
a^{3}=\frac{R}{K}\left[F+3 \pi W_{132} R+\sqrt{6 \pi W_{132} R F+\left(3 \pi W_{132} R\right)^{2}}\right]
$$

where ' $a$ ' is contact radius, ' $R$ ' is effective radius, ' $F$ ' is the force that tip pressed to the sample surface (contact load) and ' $W_{132}$ ' is the adhesion energy between two particles. And ' $K$ ' is effective young's modulus of elastic constant $[9,2]$ is,

$$
\frac{1}{K}=\frac{3}{4}\left[\frac{1-\boldsymbol{v}_{\text {samp }}^{2}}{E_{\text {samp }}}+\frac{1-\boldsymbol{v}_{\text {tip }}^{2}}{E_{\text {tip }}}\right] \text {, where ' } E \text { ' and ' } v \text { ' are }
$$

Young's modulus and Poisson's ratio.

In an extensive contact between the tip and the sample, the area of contact will be less [10] than that given by $\pi R^{2}$. For such a case the contact radius is estimated by considering, neglecting the contribution of ' $F$ ' being either small or not applicable, then the contact radius ' $a$ ' [2] is

$\left.a=\llbracket\left(\frac{3}{2} \pi R^{2} W_{132}\right) / K\right]^{\frac{1}{3}}$

This results the contact radius. Then, we know number of atoms in micro-contact. There could be number of techniques by which one can estimate number of molecules or atoms during AFM micro-contact (i.e., a spherical tip in contact with flat sample). if we take diameter of the mean atomic circumference found in the crystalline state, so that its circumference of the contact region is $C$, where $C=2 \pi a$, and let $a_{0}$ be the radius of each silicon atom, such that the number of silicon atoms that are in contact with the tip can be estimated as

$$
2 \pi a=n \cdot 2 \pi a_{0} \Rightarrow n=\frac{a}{a_{0}} \text {. }
$$

Based on this calculation we have estimated the surface energy, work of adhesion, single atomic interaction and hence net interaction strength for given pull-off force contribution. The estimated parameters are in Table 1 below

Originally (i.e., without pressure case) membrane surface have almost uniform ion distribution over the entire region as shown in net interaction strength/ surface energy calculations and distribution of pull-off force shown in Fig.3 (a). But when the membrane is pressurized, an important electrical phenomenon takes place. is being happen. By considering an electrical potential at the surface layer $\left(U_{0}\right)$ is decreases as one proceeds inside from surface layer. At any point the potential ' $U$ ' determines the potential energy ' $z e U$ ' of an ion in the local field inside, where ' $z$ ' is the valence of ion and ' $e$ ' is its charge. The probability of finding an ion at any point depends on the local potential $(U)$ through Boltzmann distribution

$\exp \left(-\frac{z e U}{k T}\right)$ 
Our assumption here is, in a strained membrane the unit cell suffers distortion and produces small displacement relative to each other. If the unit cell lacks a center of symmetry, i.e., displacement of ions (centroid of the charge shift) causes an electrostatic force. Concentration of ions that contribute electrostatic force in the diffuse layer, $n(x)$, at distance ' $x$ ' from the surface, will be in equilibrium to bulk concentration, $n_{0}$, should follow Boltzmann relation [6]

$$
n(x)=n_{0} e^{-\cdot \frac{z e U}{k T}}
$$

The potential is related to the charge density at ' $x$ ' by Poisson's equation. On solving Poisson-Boltzmann equation one can get several solutions [for details see ref 12]. Among those, solution related to surface charge density $\left(\sigma_{0}\right)[12]$ is

$\sigma_{0}=\left[2 k T \varepsilon \varepsilon_{0} \sum n_{0}\left(e^{-\frac{z e U}{k T}}-1\right)\right]^{\frac{1}{2}}$

This is the relation between surface potential and surface charge density. Where $\varepsilon_{0}$ is the permitivity of vacuum and $\varepsilon$ is the dielectric constant of the medium. Further, from the same reference

$$
\sigma_{D}=-\varepsilon \varepsilon_{0} \frac{d U_{D}(x)}{d x}
$$

Where $\sigma_{n}\left(x\right.$ and $U_{n}(x$ are surface charge density and surface potential at ' $x$ ' when the surfaces are at a distance $D$ apart. This equation clearly indicates that force and charge density are inter-related.

Hence, AFM force spectroscopy provides information that is critical to understand potential energy surface through the measurement of force (i.e., pull-off force) profile.

Our experimental results shown in Fig 3a shows that the distribution of pull-off force is almost steady in the case of without pressure condition. On the other hand, for a sufficiently thin silicon membrane that undergoes beyond the critical applied pressure, get strained layer by layer. As a result strained layer thickness increases. As the strained layer thickness increases, the total number of atoms under strained or the distorted atoms grow up and at some point lattice-mismatch is occurring which is reflected out as a localized states of the charges and hence the charge asymmetry.
Table 2 : Estimation of single atomic interaction. The net interaction strength of strained silicon membrane as

\begin{tabular}{|c|c|c|c|c|c|c|c|}
\hline \multirow[t]{3}{*}{$\begin{array}{l}\text { Micro } \\
\text { Cont. } \\
\text { (spec.) }\end{array}$} & $\begin{array}{l}\text { pull- } \\
\text { of } \\
\text { force }\end{array}$ & $\begin{array}{l}\text { work } \\
\text { of } \\
\text { adhes. }\end{array}$ & $\begin{array}{c}\text { surface } \\
\text { energy } \\
(\gamma)\end{array}$ & $\begin{array}{l}\text { conta. } \\
\text { diam- } \\
\text { meter }\end{array}$ & $\begin{array}{l}\text { no of } \\
\text { atoms } \\
\text { in }\end{array}$ & $\begin{array}{l}\text { single } \\
\text { atomic } \\
\text { in tera. }\end{array}$ & $\begin{array}{l}\text { net } \\
\text { stren-. } \\
\text { gth }\end{array}$ \\
\hline & $(\mathrm{nN})$ & $\left(\mathrm{J} / \mathrm{m}^{2}\right)$ & $\left(\mathrm{J} / \mathrm{m}^{2}\right)$ & $(\mathrm{nm})$ & contact & $(\mathrm{nN})$ & $(\mathrm{KJ} / \mathrm{mol})$ \\
\hline & 17.10 & 0.7257 & 0.3628 & 1.810 & 08 & 2.137 & 302.392 \\
\hline \multirow[t]{2}{*}{ With } & 18.30 & 0.7766 & 0.3883 & 1.851 & 08 & 2.287 & 323.612 \\
\hline & 18.80 & 0.7978 & 0.3989 & 1.867 & 08 & 2.350 & 332.454 \\
\hline$P$ & 20.70 & 0.8785 & 0.4392 & 1.928 & 09 & 2.300 & 325.381 \\
\hline $\mathrm{R}$ & 21.00 & 0.8912 & 0.4456 & 1.937 & 09 & 2.495 & 330.009 \\
\hline E & 06.00 & 0.2546 & 0.1273 & 1.273 & 06 & 1.010 & 152.488 \\
\hline S & 06.60 & 0.2801 & 0.1400 & 1.322 & 06 & 1.148 & 155.617 \\
\hline S & 05.30 & 0.2249 & 0.1145 & 1.229 & 06 & 0.991 & 124.965 \\
\hline $\mathrm{U}$ & 06.27 & 0.2661 & 0.1330 & 1.300 & 06 & 1.110 & 147.836 \\
\hline \multirow[t]{2}{*}{$\mathrm{R}$} & 05.80 & 0.2461 & 0.1230 & 1.267 & 06 & 1.053 & 136.754 \\
\hline & 05.89 & 0.2499 & 0.1249 & 1.273 & 06 & 1.064 & 138.876 \\
\hline $\mathrm{E}$ & 19.00 & 0.8063 & 0.4031 & 1.874 & 08 & 2.333 & 335.991 \\
\hline \multirow[t]{4}{*}{ S } & 20.70 & 0.8785 & 0.4392 & 1.928 & 09 & 2.471 & 325.381 \\
\hline & 21.10 & 0.8955 & 0.4477 & 1.940 & 09 & 2.502 & 331.668 \\
\hline & 20.30 & 0.8615 & 0.4307 & 1.915 & 09 & 2.439 & 319.093 \\
\hline & 16.60 & 0.7045 & 0.3522 & 1.792 & 08 & 2.131 & 301.527 \\
\hline With- & 04.60 & 0.1952 & 0.0976 & 1.173 & 05 & 0.9021 & 130.152 \\
\hline \multirow[t]{2}{*}{ Out } & 05.10 & 0.2164 & 0.1082 & 1.214 & 05 & 0.9666 & 144.299 \\
\hline & 04.81 & 0.2041 & 0.1020 & 1.191 & 05 & 0.9275 & 136.094 \\
\hline PR & 05.03 & 0.2134 & 0.1067 & 1.208 & 05 & 0.9578 & 142.318 \\
\hline ES & 04.96 & 0.2105 & 0.1052 & 1.203 & 05 & 0.9487 & 140.338 \\
\hline SU & 04.65 & 0.1973 & 0.0968 & 1.177 & 05 & 0.9086 & 131.567 \\
\hline \multirow[t]{2}{*}{$\mathrm{RE}$} & 05.12 & 0.2172 & 0.1086 & 1.215 & 05 & 0.9693 & 137.128 \\
\hline & 04.83 & 0.2049 & 0.1024 & 1.190 & 05 & 0.9321 & 136.660 \\
\hline
\end{tabular}
measured by AFM

Note: Data reported from ref [11] and used in calculations are:

$R=5 \mathrm{~nm},(\mathrm{a} / \mathrm{c}$ AFM catalogue $\mathrm{R}$ is $5-10 \mathrm{~nm}, \mathrm{I}$ take $5 \mathrm{~nm})$

$N_{A}=6.02 \times 10^{23}$ atoms $/$ mole - Avogardo's number

$a_{0=} 117 \mathrm{pm}$-atomic radius of silicon

$L=235 \mathrm{pm}-$ bond length of silicon

$E=165 \mathrm{GPa}$ - young's modulus of silicon

$v=0.22$ Poisson's ratio of silicon

Si-Si covalent bond strength $=230 \mathrm{KJ} / \mathrm{mol}$ 


\section{SURFACE ENERGY CHARACTERIZATION}

Based on our experimental data in Table1 above, pulloff force and surface energy have significant correlation. So, let's briefly discuss about it. Properties that happen over the surface are associated with surface energy. It is obvious that the coordination of surface atom is markedly changed in the presence of applied stress. Molecules on the surface layer have lower coordination number because of the lack of neighbors on one side. So, the molecules on the surface layer have higher energy than those in the interior [13]. This extra energy is surface energy and it is the sum of the energies of the broken bonds. Thus the physical surface of a crystal contains atoms with uncompensated bonds producing the surface energy [14]. Hence, in AFM experiment surface energy is the measurement of the strength of the intra-atomic forces. In addition to this, any defect in the crystal has associated surface energy with it. Higher the surface area of the defect, the higher will be the energy stored in it. A high concentration of the defects therefore more energy. Keeping this in mind, pull-off force and hence surface energy depends on highly poor molecule (defected) that are strongly attached to atoms or molecule in Si-membrane. Therefore, we conclude that surface energy is more where pull-off force is more and vice versa.

\section{Roughness characterization}

The surface, normally flat in the absence of pressure where as with pressure it is no longer flat as shown in Fig 2, is characterized now by a small depression at the center all along the Y-axis.

Height profile measurements along the line as shown in images in Fig 2a and $2 \mathrm{~b}$ are given in Fig $3 \mathrm{~b}$. It is clear from the graph that the rms height of the irregularities is $6 \mathrm{~nm}$ where as they stand only $3 \mathrm{~nm}$ after application of pressure and are dispersed all over the membrane area. Hence, the average roughness of the irregularities after pressure is at least $50 \%$ less. The detail of the height profile data for a particular line (as in Fig.2 a/b) and entire area of each scan in Fig2 are shown in Table 2 . The dominant result here is that the surface of the sample is no longer flat over several nanometers after the membrane has been deformed following the application of pressure but the average roughness is appreciably come down. It means pressure is the factor by which one can get asperities on plane surface.
Table 2: AFM data of roughness for area and line analysis

\begin{tabular}{|c|c|c|c|c|c|c|c|c|c|c|c|}
\hline \multirow{2}{*}{\multicolumn{2}{|c|}{$\begin{array}{l}\text { Sam- Specif- } \\
\text { ple ication }\end{array}$}} & \multirow{2}{*}{$\begin{array}{l}\text { scan- } \\
\text { size }\end{array}$} & \multicolumn{4}{|c|}{ Area measurement (in $\mathrm{nm}^{2}$ ) } & \multicolumn{5}{|c|}{ Line measurement (in $\mathrm{nm}$ ) } \\
\hline & & & $\mathrm{R}_{\mathrm{a}}$ & $\mathrm{R}_{\mathrm{ms}}$ & $\mathrm{Z}$ & $\mathrm{Z}_{\text {max }}$ & $\mathrm{R}_{\mathrm{a}}$ & $\mathrm{R}_{\mathrm{p}}$ & $\mathrm{R}_{\mathrm{pm}}$ & $\mathrm{R}_{\mathrm{t}}$ & $\mathrm{R}$ \\
\hline $\mathrm{Si}$ & wo & $1 \mu \mathrm{m}^{2}$ & 69 & 5.858 & 25.68 & 51.955 & 5.20 & 12.44 & 6.14 & 21.88 & 12. \\
\hline S1 & & $1 \mu \mathrm{m}^{2}$ & 2.437 & 2.938 & 11.45 & 27.787 & 1.97 & 05.61 & 1.96 & 09.97 & 3.56 \\
\hline
\end{tabular}

Where wop is without pressure and wp is with pressure

\section{CHARACTERIZATION}

In order to better understand the charge asymmetry over the extended silicon membrane, we further proceed for electrical measurements. Current-voltage data as taken from STM mode from both without/with pressure conditions of the same silicon membrane are display in Fig3c. Our experimental results on IV show that membrane under pressure almost loses its conductivity. We have the following explanation why this actually happen.

Real crystal silicon has preexisting impurities whose sizes are different than the host silicon atoms. An impurity ion, if placed into the crystal lattice, the impurity ion distort the region around it either by pushing the host atoms further away or by pulling them in [15]. Such a region becomes further enlarged and activated, as the membrane is influenced by external pressure simply because of enormous increase of dislocation density. As for example, deformation causes a great increase in dislocation density typically from $10^{8}$ to about $10^{11}$ dislocations $/ \mathrm{cm}^{2}$ during deformation [16].

If an electron is allowed to fall on distorted lattice zone, (elastic), it scatters. This is because an electron experiences an unexpected change in potential energy $(U)$ as it approaches the distorted zone. The magnitude of force that the electron suddenly experiences in this zone is, $F=-d U / d x$ due to sudden change in potential energy. Since, induced distortion extend a number of atomic distances (i.e., in multiplicative terms), as a result, distorted zone becomes larger and larger. Hence, the impurity ion therefore hinders the motion of the electrons. conduction electron as such is actually subjected to two forces: an electric force and an opposing force. Precisely speaking, the conduction electron interacts with the lattice vibrations (phonons) and with lattice imperfections (ion centers). All these factors combine to establish a definite mean free time of the electron $(t)$. As a result of the interaction a frictional force $\left(F_{R}\right)$ applied to electron is generated. Electron's velocity (i.e., drift velocity) decreases in accordance with

$v=v_{0} e^{-\tau / t}$ where $v_{0}$ is the velocity at $t=0$

If the electron's mean free time is identified with the 
relaxation time $(\tau)$, its mean velocity $(\mathrm{v})$ will be ceased to act (at $t=0$ ). Because drift velocity is generated by the frictional force and it will be minimum if there is no external force (i.e., applied pressure). In this connection, contribution of the drift velocity to the current (i.e., current density) for the conduction carriers whose charge (q), density ( $n)$ and mean drift velocity ( $v)$, would be

$$
J=n q v=n q(\mu E)=(n q \mu) E=\sigma_{c} E
$$

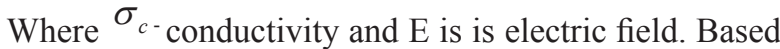
on this discussion we can say $\sigma_{c} \leq \sigma_{c n}$, where $\sigma_{c 0}$ is the conductivity in the case when there is no external pressure. This inequality is clearly reflecting the experimental results of IV curves given in Fig 3c. Hence, current contribution under pressurized case should be significantly less.

\section{XRD CHARACTERIZATION}

In order to understand the distortion that creates by applied pressure, we have performed XRD experiment. XRD pattern over the extended and un-extended silicon membrane, the data are collected and displayed in the Fig. 3d. Data retrieved from experimental XRD plot is shown in Table 3 below. In Fig $3 d$, there is noticeable change in the graph, which is, significant shift of peak position in (004) plane. This is the clear indication that external pressure is going to affect the crystallographic plane. The quantitative magnitude of distorted contraction of the plane is estimated in terms of interplanar spacing. The change in interplanar distance in particular (004) plane is $0.00384{ }^{\circ} \mathrm{A}$, can be estimated just by differentiation of Bragg's equation

$$
\begin{aligned}
& \frac{\Delta a}{a}=\frac{\Delta d}{d}=-\Delta \theta \cdot \cot \theta, \quad[\text { for constant } \lambda] \\
& \Delta d=d_{1}-d_{2}=d \cdot \Delta \theta \cdot \cot \theta=\frac{\lambda}{2 \sin \theta_{1}}-\frac{\lambda}{2 \sin \theta_{2}}
\end{aligned}
$$

where $\theta_{1}, \theta_{2}$ and $\Delta \theta$ are the angular positions on pressurized and un-pressurized x-ray spectra and their difference.

Table 3: Data retrieve from XRD spectra

\begin{tabular}{lllllll}
\hline Sam & Spec & $2 \theta\left({ }^{0}\right)$ & $\mathrm{d}\left({ }^{0} \mathrm{~A}\right)$ & \multicolumn{2}{c}{$[\mathrm{hkl}]$} \\
$\mathrm{Si} \quad$ wop & 28.46 & 69.02 & 3.13 & 1.36 & 111 & 400 \\
$\mathrm{Si} \quad$ wp & 28.04 & 69.72 & 3.14 & 1.34 & 111 & 400 \\
Calculated data & & & & & & \\
Silicon & 28.51 & 69.31 & 3.12 & 1.35 & 111 & 400 \\
\hline
\end{tabular}

Since the membrane consist of several crystallographic planes separated by, $d_{h k l}$. A membrane that deflects under applied external pressure creates contraction of the plane spacing. As a result there is a shifting in angular distance of the XRD spectra and diffraction intensity; both are easily observable experimental parameter as shown in Fig.3d. Hence, application of pressure induces a structural distortion with their twist in symmetry of the lattice.

\section{CONCLUSION}

In solid-state physics, we avoid the surface that arises unless a specific interest. Properties that happen over the surface are associated with surface energy. Under the thermodynamic equilibrium, a crystal acquires the structure that satisfies the requirement of minimum total surface energy. In a discrete lattice system on the surface of the crystal vibrates in the form of waveform. The vibratory motion of surface atoms contributes to surface free energy [13]. It is obvious that the coordination of surface atom is markedly changed in the presence of applied stress as well as crystal imperfections. Where as in inner layers the atoms are in condensed phase, the molecules are coordinated on all sides. It is obvious that the molecules on the surface have lower coordination number because they lack neighbors on one side. So that, the molecules on the surface layer have higher energy than those in the interior [14]. This extra energy is surface energy and it is the sum of the energies of the broken bond. Thus the physical surface of a crystal contains atoms with uncompensated bonds producing the surface energy [13]. Hence, surface energy is the measures of the strength of the intra-atomic forces.

We have experimentally proved that pressurized silicon membrane has pull-off force variation over the strained si-membrane that is because surface energy varies over the strained silicon membrane. This can be stated here without any doubt, based on pull-off force (i.e., measures by eq (1) and (2), variation with corresponding topography. It means there is heterogeneous distribution of force with distance. So, it is obvious that there is asymmetrical charge distribution over the strained silicon membrane.

\section{REFERENCES}

1. Marton, R. (ed). 2000. Nanosurface Chemistry. first edition Marcel Dekker Inc NY, USA.

2. Hajime, T., Jeremy, R. K., Sze-shun, W., Janese, C., O’brien. and Marc, D. Porter. 1999. Chem. Rev. 99: 2845.

3. Wang, C.Z., Pan, B.C. and Ho, K.M. 1999. J. Phys. Condens. Matter. 11:2043.

4. Berger, C.E.H., Van Der Werf, K.O., Koyman, R.P.H., Grooth De, B.G. J. and Greve, 1995. Langmuir 11: 4188.

5. Sinanoglu, O. 1981. J. Chem. Phys. 75: 463.

6. Henry, W. and Jacques O, 1990. Material Concepts in Surface Reactivity and Catalysis, Academic Press, Inc 19.

7. Cappella, B. and Dietler, G. 1999. Surface Science Report 34: 1.

8. Fuqian Yang, 2003. J. Phys D: Appl. Phys. 36:2417.

9. www.igahpse.epfl.ch 
10. Blakey, J.M. (ed). 1975. Surface Physics of Materials. Academic Press. London 497.

11. www.users.ox.ac.uk/ mwalter/web04/resources/sil_chem/ org_silicon.chem.shtml, www.ai.mit.edu/people/tk/tks/silicon. mechaniccal.

12. Frobeg, J.C., Rojas, O.J. and Claesson, P.M. 1999. Int. J. Miner. Process. 56:01.

13. Van Vlack. 1970. Material Science for Engineers, AddisonWesley Publishing Company, Reading, Massachusetts.

14. Mayer, H.P. 1998. Introduction to Solid State Physics, Viva Books Pvt Ltd. New Delhi 426.

15. Kasap, S.O. 2002. Principle of Electronic Materials and Devices, MacGraw Hill .

16. Kittel, C. 1996. Introduction to Solid State Physics, John Wiley and Sons, Singapore.p: 587. 